\title{
BMJ Open Factors related to the adoption and adherence of physical activity mobile applications by older people: a scoping review protocol
}

\author{
Lorena Jorge Lorenzi (D) , ${ }^{1}$ Letícia Fernanda Belo, ${ }^{1}$ David Mark Frohlich, ${ }^{2}$ \\ Victor Zuniga Dourado, ${ }^{3}$ Paula Costa Castro, ${ }^{1}$ Grace Angélica de Oliveira Gomes ${ }^{1}$
}

To cite: Lorenzi LJ, Belo LF, Frohlich DM, et al. Factors related to the adoption and adherence of physical activity mobile applications by older people: a scoping review protocol. BMJ Open 2021;11:e052414. doi:10.1136/ bmjopen-2021-052414

- Prepublication history and additional supplemental material for this paper are available online. To view these files, please visit the journal online (http://dx.doi.org/10.1136/ bmjopen-2021-052414).

Received 15 April 2021 Accepted 27 September 2021

Check for updates

(c) Author(s) (or their employer(s)) 2021. Re-use permitted under CC BY-NC. No commercial re-use. See rights and permissions. Published by BMJ.

${ }^{1}$ Department of Gerontology, UFSCar, Sao Carlos, Brazil ${ }^{2}$ Department of Music and Media, University of Surrey, Guildford, UK

${ }^{3}$ Department of Human Movement Science, UNIFESP, Santos, Brazil

Correspondence to Lorena Jorge Lorenzi; Iololorenzi@hotmail.com

\section{ABSTRACT}

Introduction Ageing is a natural process marked by physiological changes and declines in functional capacity. One strategy to encourage healthy habits in older people is the use of applications on mobile devices to promote physical activity (PA). An immediate challenge is for these applications to be accessible to older people themselves, while a second challenge is to retain their interest and engagement in connection with PA itself. Therefore, the purpose of this review is to map the factors related to the adoption and adherence of PA mobile applications by older people.

Methods and analysis Five databases will be searched where articles and reviews, available between 2010 and present, in English, Portuguese or Spanish, at full text, will be included. In addition, two additional strategies will be performed, including grey literature. The search terms adoption, adherence, factors, mobile application, PA, older people and other terms related to them will be used in the search strategy. This review will include studies that identify factors related to the adoption and adherence to PA mobile applications by people over 60 years. The selection of studies will be carried out by two reviewers in five stages: identification of studies and duplicate removal; pilot test; selection by reading abstracts; inclusion by reading the full text and search in additional sources. Disagreements will be resolved by a third reviewer. Data will be extracted using a data extraction tool. Quantitative data will be described in a narrative manner and qualitative data will be categorised through inductive thematic analysis.

Ethics and dissemination Ethical approval is not required for this scoping review. Plans for the dissemination of the review include the presentation of the results at relevant scientific conferences and the submission and publication in significant journals.

\section{INTRODUCTION}

Ageing is a constant and natural process, marked by physiological changes and declines in functional capacity. When associated with an unhealthy lifestyle, it can cause the development or aggravation of chronic noncommunicable diseases, which are one of the main causes of hospitalisations and deaths
Strengths and limitations of this study

- Five databases will be consulted, as well as two additional strategies, including the grey literature.

- Double-blind screening done by two reviewers independently, all abstracts and full texts will be read by both of them.

- We verified studies in two other languages besides English.

- Other studies involving older participants but do not have specific data from this population on the adoption or adherence of physical activity mobile applications will not be included.

- A limitation is the lack of critical appraisal of study quality and risk of bias assessment using this type of review.

of the older people. Some risk factors that can cause the progression of these diseases are inadequate nutrition, smoking, excessive consumption of alcoholic beverages, obesity and physical inactivity. ${ }^{1-4}$

According to Piercy et $a l^{5}{ }^{5}$ regular physical activity (PA) can help to prevent these diseases and can generate other benefits for older people such as reduce the risk of mortality from all causes, improve quality of life and cognition, decrease anxiety and risk of depression, improve sleep and bone health and reduce risk of falls and dementia. Despite these benefits, few older people are adept at the practice of PA and reach the recommended minimum. This is due to barriers such as health problems, fear of practising, lack of guidance, stimulation and motivation, difficulty in getting to facilities and places to perform. $^{6-8}$

One strategy to circumvent some of these barriers and promote an increase in PA is the use of applications on mobile devices such as e-health and m-health. ${ }^{9}$ Studies demonstrate that these applications are effective 
for increasing PA levels, motivating users to practice and changing attitudes, beliefs and perceptions. ${ }^{111}$ The use of mobile devices can generate several benefits for older people such as providing social interaction, intergenerational relationships, leisure and digital inclusion, in addition to helping to acquire knowledge and information, improve quality of life and reduce loneliness. ${ }^{12}{ }^{13} \mathrm{In}$ addition, studies point out that mobile applications are an economical alternative since most of these tools are free and affordable and the use of mobile devices by older people is growing. Another important feature of applications is offering feedback and setting goals which favours motivation. ${ }^{1415}$

However, despite the benefits of these technologies, older people may face a number of challenges in using them, such as anxieties and fears, deficits in cognitive skills, health problems, lack of instructions and guidelines and the cost. ${ }^{16}{ }^{17}$ In addition to these challenges, there are other factors that can influence individual behaviour which according to Sallis et $a l,{ }^{18}$ can be biological, psychological, familiar, occupational, recreational, political and environmental. This was verified in a study which showed that individual characteristics such as sex, age, education, health deficiencies, anxiety, self-efficacy and environmental factors such as accessibility, help and guidance, can affect the acceptance of older people to technology. ${ }^{19}$

Knowing the aspects that can influence the use of mobile devices applications by older individuals it is important to consider the demands, contexts and desires of these users in the development of more accessible technologies. ${ }^{17}{ }^{20-22}$ It is, therefore, necessary to carry out a literature review to analyse all factors that may be related to the use of these applications by them, including adoption, defined as the beginning of a practice such as downloading an application, and adherence, defined as the continuity of a practice for a period of time such as continuing to use an application. ${ }^{23}$

A preliminary search of the Cochrane Database of Systematic Reviews, Joanna Briggs Institute (JBI) Evidence Synthesis and the PubMed database was carried out to explore the feasibility and novelty of such a review. The search revealed that the review is original. Only three articles with similar themes were found, and they differ from the present review in a number of ways. For example, they do not obtain results from the older people, they do not focus exclusively on PA applications, and they only include randomised controlled clinical studies. ${ }^{24-26}$ Therefore, this scoping review will be the first to map specific factors of the older population relating to the adoption or adherence of PA mobile applications across a range of study types, based on existing literature.

Besides being original, a scoping review with this theme is relevant, as PA mobile applications must be accessible to older people and it is important to check all the factors that are related to the adoption and adherence of these applications by this population so that future applications be developed according to this population and to increase its use by them. The choice of the scoping review was due to the fact that this type of review has a broad objective and can be used to identify the studies about a topic, to verify knowledge gaps and to map factors related to a certain topic. ${ }^{27}$ Thus, the objective of the present scoping review is to map what the studies present about the factors related to the adoption and adherence of PA mobile applications by older people.

\section{METHODS AND ANALYSIS}

This scoping review will be conducted in accordance with the JBI methodology for scoping reviews ${ }^{28}$ and the Preferred Reporting Items for Systematic Reviews and Meta-Analyses (PRISMA) extension for scoping reviews. ${ }^{29}$

To develop the research questions, the PCC strategy, which means Population, Concept and Context, was used. The research questions are:

1. What do studies present as factors related to the adoption of PA mobile applications by older people?

2. What do studies present as factors related to the adherence of PA mobile applications by older people?

\section{Search strategy}

The search strategy will aim to locate both published and unpublished articles and reviews. An initial limited search of PubMed and Google Scholar was undertaken to identify articles on the topic in order to verify terms related to the research question of the present review. After consulting words contained in the titles and abstracts of relevant articles, the following search terms were defined: adoption, adherence, factors, applications, PA and older people, in addition to their related terms. These terms were used to develop full search strategy for five databases, and will be used to perform the bibliographic search of the full review. They will be investigated in titles, abstracts and keywords of studies in the chosen databases. These search strategies were adapted according to the characteristics of the databases, including the use of MeSH Terms where necessary. The preliminary search strategy for Scopus is presented in table 1.

In addition, the reference list of the studies selected in the review will be screened for additional studies meeting the inclusion criteria. Another additional source for selection of studies will be a search on Google Scholar to identify studies including grey literature such as dissertations, theses and opinion papers that meet the eligibility criteria. The first 150 studies found in the whole search will be analysed for answers to the research questions. The decision to add grey literature is because some of the studies on the subject may be patents or industry/ market research as well as theses and dissertations rather than journal articles. Many applications are released and published on different venues and this scoping review aim to broaden the search to try to map all possible studies.

The databases to be searched include Web of Science (Clarivate Analytics), PubMed (NCBI), Scopus (Elsevier), Lilacs (BVS) and Scielo. All articles and review studies that meet the criteria, available between 2010 and the present, 
Table 1 Search strategy for Scopus

\begin{tabular}{ll}
\hline Search & Query \\
\hline \#1 Population & $\begin{array}{l}\text { TITLE-ABS-KEY(elder* OR "elderly population" OR "older people" OR aged OR "ageing population" OR "older } \\
\text { population" OR ageing OR geriatric) }\end{array}$ \\
\#2 Concept & $\# 2.1$
\end{tabular}

TITLE-ABS-KEY((adoption OR adhesion OR instal OR access OR download OR acceptance OR rejection) OR (adherence OR nonadherence OR "sustained use" OR abandonment OR persistence OR maintenance OR dropout OR attendance OR compliance OR non-compliance OR noncompliance) OR (factor ${ }^{\star}$ OR preference* OR characteristic ${ }^{\star}$ OR experience ${ }^{\star}$ OR determinant ${ }^{\star}$ OR attribute* OR perception ${ }^{\star}$ OR development OR barrier $^{\star}$ OR facilitator $\left.\left.{ }^{\star}\right)\right)$

\#2.2

TITLE-ABS-KEY("physical activity" OR "motor activity" OR exercise* OR "physical training" OR fitness)

$\begin{array}{ll}\text { \#3 Context } & \text { TITLE-ABS-KEY(“mobile application" OR app OR apps OR "cell phone" OR smartphone* OR "mobile devices" } \\ \text { OR m-health OR e-health) } \\ \text { \#4 } & \text { \#1 AND \#2.1 AND \#2.2 AND \#3 }\end{array}$

Limitations: published between 2010 and 21 September 2020. Languages English OR Portuguese OR Spanish. Articles OR reviews.

in English, Portuguese or Spanish, in full text, will be included. The authors of the articles will be contacted to request missing or additional data as needed. The search strategies used in all databases are described in online supplemental file 1.

\section{Study inclusion criteria}

Eligibility criteria, as well as inclusion and exclusion criteria, are described in table 2.

This review will consider studies that include people over 60 years, with any gender and ethnicities, that have reported or identified factors related to the adoption or adherence of PA mobile applications. It is not necessary that the study participants have used an application, but the studies must have identified factors related to the use of it, in addition, the application may have other functions besides PA.

This scoping review will consider for inclusion different study designs, including quantitative, qualitative and mixed-methods study designs. The studies may or may not be published. All types of published articles such as letters to the editor, editorials and similar manuscripts will be included. Studies in Portuguese, English or Spanish language will be included. This significantly broadens the review from English-only publications to include further European and South American literature, while remaining within the language competence of the reviewers. Articles from 2010 to the present will be included based on the adoption of mobile applications from their inception on feature phone and smartphone platforms. Articles where the full-text copies cannot be obtained, after great effort and exhausted all attempts by searching the databases and the journal website and contacting the corresponding authors, will not be included. In addition, political documents or technical reports will not be included.

\section{Study/source of evidence selection}

The selection of studies will take place in five stages. We are currently starting the fourth stage. In the first stage, the bibliographic search was carried out across the selected databases, according to the complete search strategy developed. All records generated by this search were exported to the Microsoft Excel 2016 program, in which the titles of the articles were placed in the same language to perform duplicate removal. To ensure the removal of all duplicates, the titles were placed in alphabetical order and two reviewers scanned the table and removed the duplicates that still existed.

In the second stage, a pilot test was carried out in which two reviewers independently screened the titles and abstracts of 25 studies found with the bibliographic search. Through this pilot test, it was possible to verify

Table 2 Eligibility criteria, inclusion and exclusion criteria of the review

\begin{tabular}{lll}
\hline Eligibility criteria & Inclusion criteria & Exclusion criteria \\
\hline $\begin{array}{l}\text { Present data about a mobile } \\
\text { technology for physical activity } \\
\text { (may have other goals as well). }\end{array}$ & $\begin{array}{l}\text { Have reported or identified factors related } \\
\text { to the adoption or adherence of PA mobile } \\
\text { applications by people over 60years }\end{array}$ & $\begin{array}{l}\text { Articles where the full-text copies cannot } \\
\text { be obtained (after great effort to get it) }\end{array}$ \\
$\begin{array}{ll}\text { Include people over 60years old. } & \begin{array}{l}\text { Studies in Portuguese, English or Spanish } \\
\text { language }\end{array}\end{array}$ & Political documents or technical reports \\
& Articles from 2010 to search date & \\
\hline
\end{tabular}

PA, physical activity. 
if the eligibility criteria are well defined. If there is $75 \%$ or more agreement between the reviewers, the selection of the studies will proceed to the third stage. If this percentage is less than $75 \%$, the eligibility criteria will be modified until this percentage agreement is achieved.

In the third stage, the two reviewers independently screened all titles and abstracts of studies found in the bibliographic search by applying the eligibility criteria to select the studies for the next stage. In the fourth stage, which is the one we are starting to perform, the two reviewers will independently read all full texts of the articles that were chosen in the third stage, in order to choose those that will be included in the study, according to the inclusion criteria. Any disagreements that arise between the reviewers during the selection process will be resolved by a third reviewer. The screening of the abstracts and the full texts, that is steps 3 and 4 , will be performed in a double-blind fashion by the two reviewers, who will read all the studies found independently without conferring. The last stage will be the realisation of the additional strategies, which are the reading of the studies that were included in the review (fourth step) and of the first 150 studies found according to the Google relevance algorithm, including grey literature. The studies will be read according to steps three and four, in order to verify whether they will be included in the review.

The reasons for exclusion of full-text papers that do not meet the inclusion criteria will be recorded and reported in the scoping review. The results of the search will be reported in the scoping review and presented in a PRISMA flow diagram. ${ }^{30}$

\section{Data extraction}

Data will be extracted from the studies included in the scoping review using a data extraction tool developed by the reviewers. This tool will be a table in Microsoft Excel 2016 called 'Data extraction form' (in the online supplemental file 2), which will be individual for each study, in order to summarise their main information. The data extracted will include specific details about the population, concept, context, methods and key findings relevant to the review research questions. This extraction form was developed based on the recommendations of the guidelines in the JBI recommendations, ${ }^{27}$ but other information has been included too. Thus, this form will contain information about study characteristics, intervention chosen for the study, characteristics of the study participants, characteristics of the mobile application used in the study, and factors related to the participant's adoption or adherence to the PA mobile application (such as barriers to download or use, reasons to download or maintain the use, reasons for withdrawal or abandonment of use, suggestions for improving or developing the mobile application, sociodemographic factors related to the use of the application and other information).

The reviewers will trial the extraction tool on two or three studies to ensure that all relevant results are extracted. This draft data extraction tool will be modified and revised as necessary during the process of extracting data from each included paper. Modifications will be detailed in the full scoping review.

\section{Data analysis and presentation}

The extracted data will be presented in diagrammatic or tabular form in a manner that aligns with the objective of this scoping review. The results will be reported through the numerical identification of the studies and the discussion of their data, including the number of studies that were not included and the main reason for rejection, the number of studies included, general characteristics of the studies, types of study designs, population, characteristics of the interventions and applications used, factors related to adoption of PA mobile applications, factors related to adherence and gaps of the research.

Two reviewers will extract data from the included articles and compare tables, resolving disagreements through discussion. Quantitative data will be described in a narrative manner and analysed using absolute and relative frequency, percentage, average and SD. To verify the factors, reviewers will conduct an inductive thematic analysis on qualitative data. Constant comparison will be used to refine emerging conceptual categories, including a search for deviant cases. We will then list all types of factors that will appear and categorise them (eg, barriers to using the app, reasons to download, reasons to continue using, sociodemographic factors related to usage), in which we will present the categories of factors that appeared in each article and, if necessary, subcategorise what was found in each category. This mapping can help guide the development of mobile apps that are more accessible to older end users. A likely date for completing all stages of the review and writing a manuscript will be October of this year.

\section{Patient and public involvement statement}

Patient and public were not involved in this study.

\section{ETHICS AND DISSEMINATION}

Due to the fact that this review will generate knowledge about published and unpublished literature in the public domain, ethical approval is not required for this scoping review. This review can identify information and gaps in the existing evidence literature. Plans for the dissemination of the review for the community include the presentation of the results at relevant scientific conferences and the submission and publication in significant journals.

Acknowledgements We acknowledge contributions from Dr Marilia M. De Almeida Cardoso, member and trainer of the JBI Brasil Center of Excellence at USP School of Nursing, to the feedback on the review protocol.

Contributors All authors have made substantial contributions to the development of the protocol. LJL conceptualised the research question and prepared the first draft of the manuscript. LJL, LFB, GAdOG and PCC contributed to the refining of the study design and guided the protocol development. LJL, LFB, GAdOG, PCC, VZD and DMF contributed to the writing, editing and revising of this protocol. DMF provided review expertise and assisted in the revision of the English. 
Funding LJL, VZD and LFB received funding from São Paulo Research Foundation (FAPESP), No 2019/02829-9, № 2016/50249-3 and No 2019/11584-0, to the development of some studies, including this review. And LJL received funding from the Coordination for the Improvement of Higher Education Personnel (CAPES) Financial Code 001

Competing interests None declared.

Patient consent for publication Not applicable.

Provenance and peer review Not commissioned; externally peer reviewed.

Supplemental material This content has been supplied by the author(s). It has not been vetted by BMJ Publishing Group Limited (BMJ) and may not have been peer-reviewed. Any opinions or recommendations discussed are solely those of the author(s) and are not endorsed by BMJ. BMJ disclaims all liability and responsibility arising from any reliance placed on the content. Where the content includes any translated material, BMJ does not warrant the accuracy and reliability of the translations (including but not limited to local regulations, clinical guidelines, terminology, drug names and drug dosages), and is not responsible for any error and/or omissions arising from translation and adaptation or otherwise.

Open access This is an open access article distributed in accordance with the Creative Commons Attribution Non Commercial (CC BY-NC 4.0) license, which permits others to distribute, remix, adapt, build upon this work non-commercially, and license their derivative works on different terms, provided the original work is properly cited, appropriate credit is given, any changes made indicated, and the use is non-commercial. See: http://creativecommons.org/licenses/by-nc/4.0/.

\section{ORCID iD}

Lorena Jorge Lorenzi http://orcid.org/0000-0002-2378-1263

\section{REFERENCES}

1 Schmidt MI, Duncan BB, Azevedo e Silva G, et al. Chronic noncommunicable diseases in Brazil: burden and current challenges. Lancet 2011;377:1949-61.

2 Malta DC, Bernal RTI, Lima MG. Doenças crônicas não transmissíveis $E$ a utilização de serviços de saúde: análise dA Pesquisa Nacional de Saúde no Brasil. Rev Saude Publica 2017;51.

3 Gama KNM, Morais RC, Oliveira V. Atividades Físicas para Idosos: motivos para aderência E permanência. LifeStyle 2019;6:51-9.

4 Martins NFF, Abreu DPG, Silva MRS. Nursing scientific production on noncommunicable diseases in the elderly: relationships with health needs, research priorities in Brazil and nursing work. Research, Society and Development 2020;9:e52932336.

5 Piercy KL, Troiano RP, Ballard RM, et al. The physical activity guidelines for Americans. JAMA 2018;320:2020-8.

6 Hauser E, Gonçalves AK, Martins VF. Motivos de desistência em um programa de atividades física para idosos. Rev Kairós Gerontologia 2014;17:43-56.

7 Baert V, Gorus E, Mets T, et al. Motivators and barriers for physical activity in older adults with osteoporosis. J Geriatr Phys Ther 2015;38:105-14.

8 Barroso Júnior JC, Faria Junior AGde. A relação entre a adesão $E$ a evasão de idosos em projetos $\mathrm{E}$ atividades físicas1. Ciência em Movimento 2017;19:73-82.

9 Ferrara G, Kim J, Lin S, et al. A focused review of smartphone diet-tracking apps: usability, functionality, coherence with behavior change theory, and comparative validity of nutrient intake and energy estimates. JMIR Mhealth Uhealth 2019;7:e9232.

10 Fanning J, Roberts S, Hillman $\mathrm{CH}$, et al. A smartphone "app"delivered randomized factorial trial targeting physical activity in adults. J Behav Med 2017;40:712-29.
11 Hoj TH, Covey EL, Jones AC, et al. How do apps work? an analysis of physical activity APP users' perceptions of behavior change mechanisms. JMIR Mhealth Uhealth 2017;5:e114.

12 Santos DB, Feitosa ET, Silva RO. O uso de tecnologias pela população idosa brasileira. Tecnologias em Projeção 2016;7:80-7.

13 Casadei G, Bennemann R, Lucena T. Influência das redes sociais virtuais na saúde dos idosos. Enciclopédia Biosfera 2019;16:1962-75.

14 Edney S, Ryan JC, Olds T, et al. User engagement and attrition in an app-based physical activity intervention: secondary analysis of a randomized controlled trial. J Med Internet Res 2019;21:e14645.

15 Yerrakalva D, Yerrakalva D, Hajna S, et al. Effects of mobile health APP interventions on sedentary time, physical activity, and fitness in older adults: systematic review and meta-analysis. J Med Internet Res 2019;21:e14343.

16 Mostaghel R, Oghazi P. Elderly and technology tools: a fuzzyset qualitative comparative analysis. Qual Quant 2017;51:1969-82.

17 Vaportzis E, Clausen MG, Gow AJ. Older adults perceptions of technology and barriers to interacting with tablet computers: a focus group study. Front Psychol 2017;8:1687

18 Sallis JF, Cervero RB, Ascher W, et al. An ecological approach to creating active living communities. Annu Rev Public Health 2006;27:297-322.

19 Chen K, Chan AHS. Gerontechnology acceptance by elderly Hong Kong Chinese: a senior technology acceptance model (STAM). Ergonomics 2014;57:635-52.

20 Brodie MA, Pliner EM, Ho A, et al. Big data vs accurate data in health research: large-scale physical activity monitoring, smartphones, wearable devices and risk of unconscious bias. Med Hypotheses 2018;119:32-6.

21 van Beukering $M$, Velu $A$, van den Berg $L$, et al. Usability and usefulness of a mobile health APP for pregnancy-related work advice: mixed-methods approach. JMIR Mhealth Uhealth 2019;7:e11442.

22 Griffin L, Lee D, Jaisle A, et al. Creating an mHealth APP for colorectal cancer screening: user-centered design approach. JMIR Hum Factors 2019;6:e12700.

23 Telles TCB, Araruna LC, Almeida MS, et al. Adesão e aderência ao exercício: um estudo bibliográfico. Revista Brasileira de Psicologia do Esporte 2016;6:109-20.

24 Kourbelis C, Franzon J, Foote JW, et al. Adherence to activity monitoring devices or smartphone applications for improving physical activity in adults with cardiovascular disease: a systematic review protocol. JBI Database System Rev Implement Rep 2018;16:1634-42.

25 Yang X, Ma L, Zhao X, et al. Factors influencing user's adherence to physical activity applications: a scoping literature review and future directions. Int J Med Inform 2020;134:104039.

26 Ahmad NA, Mat Ludin AF, Shahar S, et al. Willingness, perceived barriers and motivators in adopting mobile applications for healthrelated interventions among older adults: a scoping review protocol. BMJ Open 2020;10:e033870.

27 Munn Z, Peters MDJ, Stern C, et al. Systematic review or scoping review? Guidance for authors when choosing between a systematic or scoping review approach. BMC Med Res Methodol 2018;18.

28 Peters MDJ, Godfrey C, Mclnerney P. Chapter 11: scoping reviews (2020 version). In: Aromataris E, Munn Z, eds. Joanna briggs institute reviewer's manual, 2020. https://reviewersmanual.joannabriggs.org/

29 Tricco AC, Lillie E, Zarin W, et al. PRISMA extension for scoping reviews (PRISMA-ScR): checklist and explanation. Ann Intern Med 2018;169:467-73.

30 Moher D, Liberati A, Tetzlaff J, et al. Preferred reporting items for systematic reviews and meta-analyses: the PRISMA statement. PLoS Med 2009;6:e1000097. 